\title{
LA FORMACIÓN DE PROFESSORES EN ESPAÑA
}

\author{
Teachers training in Spain
}

\author{
Maria Teresa González Astudillo
}

Profesora del Departamento de Didáctica de la Matemática y Didáctica de las Ciencias Experimentales de la Universidad de Salamanca (España). Vicedecana de la Facultad de Educación, Salamanca - Espanha, e-mail: maite@usal.es

\section{Resumen}

En España, la formación de los profesores ha seguido distintos modelos a lo largo de la historia, condicionados por la situación política y social de cada momento. Desde la creación de la primera Escuela Normal hasta la revisión actual de los planes de estudio para adaptarlos al contexto europeo, en este escrito se revisa el devenir de esta formación.

Palabras-clave: Formación de profesores; Legislación; Planes de estudio.

\section{Abstract}

In Spain the teachers training has followed different models along the history conditioned by the political an social situation in each moment. From the creation of the first Norma School until the actual revision of the curriculum in order to adapt the curriculum to European context, in these pages the development of this training is revised.

Keywords: Teachers training; Legislation; Curriculum. 
Desde la creación y consolidación del Sistema Nacional de Educación en España, a mediados del siglo XIX, se puede asegurar que han existido dos modelos en la Formación del Profesorado en España. El primero de ellos se refiere a la Formación del Profesorado de Educación Primaria ${ }^{1}$ en instituciones específicas, que han recibido distintas denominaciones a lo largo de la historia: Escuelas Normales, Escuelas de Magisterio, Escuelas Universitarias de Formación del Profesorado de Educación General Básica; en estas instituciones el profesor de educación primaria recibía y recibe una formación con cuatro componentes: científica, didáctica, psico-pedagógica y prácticas de enseñanza; estas cuatro componentes han tenido diferente peso a lo largo de la historia. El segundo modelo se refiere a la formación del profesorado de secundaria donde ha primado ante todo la formación científica en las Facultades Universitarias con algún añadido en formación psicopedagógica y en didácticas especiales.

\section{Evolución histórica de la formación de los profesores de primaria}

La Formación Inicial de Profesores de Primaria en España, entendida de forma institucionalizada, tiene una antigüedad de más de ciento cincuenta $a_{n}{ }^{2}{ }^{2}$ en los que se han seguido diferentes planes de estudio adaptados a las exigencias socioculturales y políticas de la época en la que se implantaron. En general, a lo largo de la historia asistimos a una tensión entre los planes que podemos denominar "culturalistas" y los "profesionales"; en los primeros ha primado la formación en contenidos y en los segundos la formación en la didáctica de las distintas disciplinas y la orientación profesional (JUNTA DE CASTILLA; LEÓN, 2007).

La creación e implantación de las Escuelas Normales en España sucede paralelamente a la implantación de un Sistema Nacional de Educación gestionado por las administraciones públicas. Durante todo el siglo XIX la mayoría de los Estados Europeos establecen, entre los objetivos de su política educativa,

1 En el sistema educativo español, salvo periodos muy concretos, la denominación general y oficial del Profesor de Primaria ha sido la de Maestro.

2 En España, tenemos que remontarnos al siglo XIV para localizar antecedentes de la formación del magisterio. En 1370, una Real Cédula promulgada por Felipe II, autorizaba al Consejo de Castillo para que examinara a los maestros con la facultad de expedir los títulos que se estimaran oportunos. Durante los siglos XVII y XVIII fue la Hermandad de San Casiano, a modo de gremio, la que concedía los títulos de maestro. A finales del siglo XVIII se Carlos III constituyó el Colegio Académico de Nobles y Bellas Arte de Primeras letras y finalmente Carlos IV crea la Academia de primera Educación que son los antecedentes de lo que posteriormente se llamarían Normales.

Rev. Diálogo Educ., Curitiba, v. 8, n. 23, p. 39-54, jan./abr. 2008 
el desarrollo y control de los sistemas de escolarización de la población infantil para conseguir, por una parte, elevar el nivel cultural general necesario para el establecimiento y desarrollo de la sociedad burguesa y, por otra, para lograr la afirmación de su poder frente a los grupos que lo detentaban en el Antiguo Régimen, en particular, frente a las instituciones y órdenes religiosas. En España, las Escuelas Normales se crean después del periodo absolutista de Fernando VII, tras la toma del poder por la burguesía liberal, influida por su contacto con las clases progresistas de Francia e Inglaterra.

Los autores especializados en la historia de las Escuelas Normales en España establecen distintas etapas para el estudio de dicha historia que, adaptadas hasta el momento actual, se pueden concretar en:

- primera etapa: desde la fundación de la primera Escuela Normal en 1839 hasta la Restauración de 1875; destaca la Ley Moyano de 1857 ;

- segunda etapa: desde 1875 a 1931, Restauración; ${ }^{3}$

- tercera etapa: desde 1931 a 1936, etapa de la República;

- cuarta etapa: desde 1939 a 1975, el franquismo;

- quinta etapa: desde 1975, la etapa de la democracia.

Desde la fundación de la primera Escuela Normal hasta la Restauración.

La primera Escuela Normal o Seminario Central de Maestros del Reino se inauguró en Madrid el 8 de marzo de 1839 siendo su primer director el zamorano Pablo Montesinos; constituyó el punto inicial de la modernización en la formación del Profesorado de Primaria. El Seminario se configuraba como una institución para recibir estudiantes pensionados por sus respectivas provincias que, una vez acabados sus estudios, se encargarían de la dirección de las respectivas Escuelas Normales provinciales. El Plan de Estudios constaba de dos años y de su análisis se concluye que era eminentemente culturalista; ${ }^{4}$ incluía Prácticas de enseñanza que se llevaban a cabo en un escuela primaria aneja al Seminario (RICO; SIERRA, 1996). A partir de esta primera Escuela la apertura de nuevas Escuelas Normales se extendió por todo el país, de modo que en 1845 funcionaban ya cuarenta y dos Escuelas

3 Se conoce con el nombre de La Restauración al periodo republicano, desde 1931 hasta 1936, fecha en la que comienza la Guerra Civil Española. Algunos historiadores han considerado esta época como Edad de Plata de la cultura española, puesto que constituye una época de despegue e impulso social y cultural.

4 El currículo se componía de dos cursos y en él pesaban fuertemente los componentes culturales (lectura, escritura, lengua, matemáticas, historia natural, física, geografía e historia y religión y moral). Los conocimientos pedagógicos se cubrían con dos disciplinas: "Principios generales de educación moral, intelectual y física" y "Métodos de enseñanza y Pedagogía". A esta formación se añadían las "prácticas" que se desarrollaban en la escuela aneja al seminario.

Rev. Diálogo Educ., Curitiba, v. 8, n. 23, p. 39-54, jan./abr. 2008 
Normales en provincias y sólo siete provincias carecían de este tipo de Institución. Las nuevas instituciones se proponían superar los mecanismos tradicionales de formación y control de los maestros de primeras letras.

Entre 1843 y 1858 se consolidó un modelo de formación del profesorado claramente distinto del modelo tradicional, que tuvo que superar la oposición hacia el monopolio estatal en la enseñanza primaria. Escolano (1982) señala la reducida presencia de componentes pedagógicos y didácticos en la formación de los maestros durante este periodo, tanto en su vertiente teórica como en la práctica.

La Ley Moyano de 1857 consideró las Escuelas Normales como escuelas de carácter profesional, diferenciadas de los Institutos de Bachillerato y de las Facultades Universitarias. No obstante, el Plan de Estudios se estructuraba con una componente culturalista muy fuerte y constaba de dos cursos para el grado de Maestro Elemental, uno más para el de Maestro Superior y otro para el grado de Maestro Normal. El primer título (Elemental) habilitaba para la docencia en el Ciclo Elemental de la Enseñanza Primaria; el segundo (Superior), para el Ciclo Superior de Enseñanza; y el grado de Maestro Normal para la enseñanza en las Escuelas Normales. En 1858 se crea la Escuela Normal Central de Maestras, en donde se realizaba la formación de maestras elementales y superiores aunque las exigencias para obtener el título de maestra, eran claramente inferiores a las que regían para los maestros.

\section{Periodo de la restauración, 1875-1931}

A pesar de las críticas que el movimiento de la Institución Libre de Enseñanza $^{5}$ (ILE) y otros sectores liberales dirigieron contra los responsables ministeriales, las Escuelas Normales se vieron sumidas en un total olvido y abandono durante la primera fase de la Restauración. A finales del siglo XIX se producen reformas controvertidas: la reforma Gamazo de 1898 redujo considerablemente el periodo de formación de los maestros y la de Romanones, en 1901, incorporó las Escuelas de Maestros y Maestras a los Institutos Generales y Técnicos de Segunda Enseñanza y suprimió el grado de Maestros Normales. Sin embargo, en 1903 los estudios de Magisterio retornan a las Escuelas Normales,

5 La Institución Libre de Enseñanza o ILE, fue un famoso intento pedagógico que se realizó en España inspirado en la filosfía de Kart Christian Friedrich Krause (Krausismo) que tuvo una repercusión excepcional en la vida intelectual de la nación, en la que desempeñó una labor fundamental de renovación. Fue creada en 1876 por un grupo de catedráticos (Francisco Giner de los Ríos, Gumersindo de Azcárate y Nicolás Salmerón, entre otros) separados de la Universidad Central de Madrid por defender la libertad de cátedra y negarse a ajustar sus enseñanzas a cualquier dogma oficial en materia religiosa, política o moral. En consecuencia, tuvieron que proseguir su labor educativa al margen del Estado creando un establecimiento educativo privado laico, que empezó en primer lugar por la enseñanza universitaria y después se extendió a la educación primaria y secundaria. Este movimiento duró prácticamente hasta el inicio de la Guerra Civil.

Rev. Diálogo Educ., Curitiba, v. 8, n. 23, p. 39-54, jan./abr. 2008 
se establece la edad mínima de catorce años para poder ingresar en estos Centros y se dispone que los estudios conducentes al título de Maestro Elemental sean de dos años, y de otros dos para el título de Maestro Superior. Se observa, durante esta época, la falta de claridad en la función de las Escuelas Normales, así como la ausencia de una política coherente en la formación de Maestros.

La evolución de las Normales estuvo condicionada por su precariedad económica, los intentos de supresión o reducción, sobre todo, por motivos políticos entre los que destacan las reticencias de los gobiernos conservadores a extender la educación a toda la población.

A partir de 1907 se crea la Junta de Ampliación de Estudios que intentaba elevar la cualificación del profesorado español a través de cursos, becas para el extranjero, publicaciones, etc. Manuel Bartolomé Cossío (1957-1935) resultó un pilar importante dentro de la formación del magisterio tanto al frente del Museo Pedagógico Nacional como de la cátedra de Pedagogía Superior. El avance en la transformación del magisterio viene representado por la creación en el año 1909 de la Escuela Superior de Magisterio (1909-1932) cuya misión era formar profesores de Escuelas Normales e Inspectores de Enseñanza Primaria. En 1914 se reforma el Plan de Estudios de la Escuela Superior que pasa a denominarse Escuela de Estudios Superiores de Magisterio: Letras, Ciencias y Labores. Este centro se estableció con las innovaciones acaecidas en otros países y con un aporte fundamental de la práctica educativa de la ILE. Así, se mejoró la calidad de la enseñanza, se contribuyó a elevar la categoría educativa y se cimentaron las bases sobre las que, posteriormente, se construyó la reforma republicana.

El año 1914 es una fecha importante en la evolución histórica de las Normales merced a la reforma del Plan de Estudios llevada a cabo por el ministro F. Bergamín García. Este Plan, vigente hasta 1931 tuvo una orientación enciclopedista en detrimento de la formación profesional y las didácticas de las respectivas materias tenían un carácter residual. Se exigía un examen de ingreso y cuatro cursos en la Escuela Normal, los dos primeros teóricos y en los dos últimos alternando le pedagogía con las prácticas. La gran novedad fue la titulación única rompiendo la jerarquía entre grado elemental y superior.

Con la dictadura de Primo de Rivera la educación se trató como un medio de control de la ideología de tendencia clerical y patriótica. Durante esta época se descuida la formación de los maestros y aunque se clamaba por una renovación en la formación de los maestros, los intentos quedaron en el nivel teórico.

\section{Las escuelas normales durante la república (1931-1936)}

La tercera etapa está marcada por el plan de estudios de 1931, paradigma de Plan Profesional, conocido habitualmente con este nombre y que tuvo muy en cuenta el modelo francés al poner al sistema educativo como motor

Rev. Diálogo Educ., Curitiba, v. 8, n. 23, p. 39-54, jan./abr. 2008 
de la modernización del país (PEREYRA-GARCIA, 2003). Es la época de la República en la que se llevó a cabo un gran esfuerzo renovador dándose cita las nuevas corrientes pedagógicas junto a la reconstrucción de la pedagogía y la formación de nuevos docentes. Los republicanos asumieron la revolución a través de la educación, con una renovación de la enseñanza primaria y del magisterio, y un ambicioso plan que pretendía erradicar el analfabetismo.

Este plan de estudios ha sido valorado como el más completo que se ha publicado para las Escuelas Normales. Cubría la formación científica, pedagógica y práctica elevando la preparación cultura y pedagógica de los maestros. Por decreto de 29 de septiembre de 1931 se exige tener dieciséis años, el titulo de Bachiller Universitario y un examen de ingreso para el acceso a la Escuela Normal lo que contribuyó a elevar el nivel de los estudios. El modelo de este proyecto no era formativo sino profesional y los estudios tenían categoría universitaria, así las Escuelas de Magisterio se convirtieron en Institutos Universitarios. Merecen destacarse las siguientes características de este Plan de estudios:

- organizaba la formación de los maestros en tres periodos: Cultura general (Bachillerato en Institutos de Segunda Enseñanza), formación profesional (tres años de estudios en las Escuelas Normales) y práctica docente (un año de Prácticas en las Escuelas Primarias).

- el curriculum del Plan de Estudios se dividía en:

a) conocimientos filosóficos, pedagógicos y sociales;

b) metodologías especiales;

c) materias artísticas y prácticas.

En el tercer curso estaba prevista la organización de enseñanzas especiales de párvulos, retrasados, superdotados etc (llamados Trabajos de Especialización) así como Seminarios acerca de aquellas materias sobre las que el alumno quisiera profundizar (llamados Trabajos de Seminario). Al concluir el tercer curso había un examen final de conjunto, presidido por un Catedrático de Universidad. Se establecía la coeducación ${ }^{6}$ en las Escuelas Normales, con profesorado masculino y femenino, las prácticas remunerada y el acceso al terminar los estudios a una plaza de maestro de Enseñanza Primaria Pública.

6 Hasta este momento la formación del profesorado fue de carácter sexista coexistiendo en las mismas ciudades centros diferentes para la formación de los hombres y de las mujeres. En este momento los centros pasan a ser mixtos con los que se redujo el número de centros que pasaron de 91 (50 de maestras y 41 de maestros) a 54 centros con un total de 540 profesores.

Rev. Diálogo Educ., Curitiba, v. 8, n. 23, p. 39-54, jan./abr. 2008 
Este Plan de Estudios formó excelentes profesionales en la enseñanza, con un prestigio reconocido en toda la sociedad española; su concepción y puesta en práctica no fue en modo alguno una imposición desde el Gobierno de la Nación, sino el resultado de un amplio movimiento, que, ante todo, pretendió dar un sentido profesional cualificado a la formación de los Maestros, entendiendo que en este terreno se fundaba uno de los elementos de progreso de nuestro país.

\section{Las escuelas normales durante el franquismo (1936-1975)}

El golpe de estado del general Franco cercenó la política republicana. La enseñanza en las escuelas Normales continuó hasta 1938 pero las circunstancias socio-políticas exigían un nuevo planteamiento en la formación de los maestros.

Después de la Guerra Civil española la formación de Maestros pierde la consideración de formación universitaria que tenía en el Plan de estudios de 1931. Se promulgaron, como respuesta de emergencia a la situación creada tras la Guerra Civil, una serie de planes como: el plan de 1940, conocido como el plan Bachiller pues sólo se exigía ser bachiller, aprobar catorce asignaturas y realizar dos cursos de prácticas a quienes desearan titularse como maestros, o el plan de 1945 que exige el ingreso con cuatro cursos de bachillerato (con 14 o 15 años) y consta de tres cursos en la Normal, después del correspondiente examen de reválida y de las Oposiciones, se accedía a la docencia con 17 o 18 años. Es un plan provisional, de corta duración que se ve completado con el de 1950, donde se aprueba el reglamento de las Normales.

El plan de 1967, en pleno desarrollismo español, intenta recuperar algunas ideas del plan de 1931, requería bachillerato superior y la realización de una reválida para ingresar en las Escuelas de Magisterio. La formación que recibían los maestros se concretaba en dieciséis asignaturas repartidas en dos cursos académicos que culminaban con una prueba de madurez. Prácticamente todas las asignaturas referidas a las áreas del currículo comenzaban por "Didáctica de ..." buscando una mayor profesionalización pero, en realidad los contenidos no eran tan didácticos como pudiera parecer. En todos los casos se combinaba el estudio puro de cada ciencia con algunos aspectos de su metodología de enseñanza basada en las corrientes de la época: la matemática moderna, la lingüística estructural y la enseñanza individualizada (Madrid, 2003). La prueba de madurez consistía en una prueba sobre a) los contenidos de las materias cursadas durante la carrera, b) el desarrollo de dos temas elegidos al azar de un temario, c) un comentario de un texto pedagógico, d) un ejercicio práctico de habilidad manual y técnica y e) una traducción de un texto en inglés o francés. Los que pasaban dicha prueba iniciaban un periodo de prácticas en las escuelas, consideradas las mejores prácticas del

Rev. Diálogo Educ., Curitiba, v. 8, n. 23, p. 39-54, jan./abr. 2008 
magisterio español hasta la actualidad, de un curso de duración, con seminarios didácticos de apoyo al final de cada jornada escolar de dieciséis a dieciocho horas de duración en las diversas disciplinas para relacionar la teoría con la práctica.

\section{Etapa democrática}

En 1970, la Ley General de Educación (LGE), devolvió a la formación de Maestros su carácter universitario ya que el Real Decreto 1381/ 1972 convirtió las antiguas escuelas Normales en Escuelas Universitarias pasando a depender administrativa de una Universidad y considerándose como centros destinados a cursar el primer ciclo universitario, aunque el profesorado tardó en integrarse de forma real en la Universidad. Esta ambigüedad de las Escuelas Universitarias del Profesorado de E.G.B. (EUFP) respecto de la Universidad no desapareció hasta la consolidación de la estructura departamental de la Universidad en 1985 con la LRU (Ley de Reforma Universitaria). La función principal de las Escuelas Universitarias fue la de preparar profesionalmente a los especialistas de las distintas áreas de la E.G.B (Enseñanza General Básica, de 6 a 14 años).

Se promulgó en 1971 un Plan de estudios con carácter experimental que fue refrendado de forma oficial en la Orden Ministerial 1411/77 (BOE de 13 de junio de 1977). Los nuevos maestros diplomados debían estar capacitados para impartir una enseñanza globalizada en la primera etapa de la EGB y de la segunda etapa, en un Área de moderada especialización. Los estudios estaban organizados de la siguiente forma:

- disciplinas comunes, que preparaban para ejercer como profesor generalista en la primera etapa;

- de especialización en un área de la EGB, que se orientaba hacia la especialización moderada en Letras o Ciencias;

- optativas, que se proponían para profundizar en las especialidades;

- prácticas de enseñanza en los cursos segundo y tercero.

La situación actual de la formación de los maestros establecida en el plan de estudios de 1991 deriva de las competencias asignadas al profesor de primaria en la Ley de Ordenación General del Sistema Educativo (LOGSE). La orientación profesional, la formación práctica y la puesta a punto de nuevas especialidades (Educación Infantil, Lengua Extranjera, Educación Física, Educación Musical, Educación Especial y Audición y Lenguaje) son las claves sobre las que se configuran los títulos de este plan de estudios que en cada una de las titulaciones de magisterio tiene un total de 200 créditos asignados (2000 horas repartidas en tres cursos académicos). Esta carga docente se distribuye en

Rev. Diálogo Educ., Curitiba, v. 8, n. 23, p. 39-54, jan./abr. 2008 
asignaturas troncales (establecidas a nivel nacional), obligatorias de universidad (establecidas por cada universidad), optativas (ofertadas por los departamentos para cada título) y créditos de libre configuración (ofertados por los departamentos para toda la universidad). Los créditos asignados a la troncalidad (120) suponen el $60 \%$ de la carga lectiva global.

El principal problema de esta estructura es que hay "títulos universitarios de maestro diferentes para idénticas responsabilidades en las áreas básicas del currículum" (Maldonado, 2005). En algunas especialidades y universidades, la formación generalista del maestro está bajo mínimos y en la práctica estos maestros especialistas deben cubrir la enseñanza de las áreas básicas que, curiosamente, en algunos casos nunca han cursado.

La transformación de la Diplomatura ${ }^{7}$ de Magisterio en Licenciatura ya se propuso por parte del Grupo XV, que elaboró los planes de estudios que están vigentes en la actualidad pero que no fue aceptada por la Administración. Lo que sí se produjo en las diferentes universidades (salvo casos aislados) fue la desaparición de las EUFP y la integración junto con los estudios de Pedagogía en las actualmente denominadas Facultades de Educación

\section{Formación inicial de los profesores de secundaria}

Desde el inicio del Sistema Nacional de Educación, durante la primera mitad del siglo XIX, ha habido intentos fallidos de dotar de una formación didáctica y profesional, además de la formación científica, a los Profesores de Enseñanza Secundaria (o Enseñanza Media, como se llamaba entonces). Como comenta Puelles (2004):

"no ha existido a lo largo de casi doscientos años una institución específica para la formación de profesores de secundaria, [éstos] sólo han sido licenciados universitarios en una determinada carrera con un ligero baño de formación pedagógica" El Instituto-Escuela (1918-1932) fue, sin lugar a dudas, el intento más claro de impartir dicha formación.

Los profesores de educación secundaria en España, hasta el momento actual, son licenciados en las diferentes ramas del currículo (Física, Matemáticas, Lengua, Geografía, Griego,...) que reciben al finalizar sus estudios un curso de formación pedagógica; C.A.P. (Curso de Aptitud Pedagógica) que data del año 1970 (GONZÁLEZ; PINTO, 2006). Esta formación dura ya más de treinta y cinco años inamovible. Ya en la ley 14/1970 de 4 de agosto (B.O.E.

\footnotetext{
En España las diplomaturas son estudios universitarios de tres años de duración, mientras que las licenciaturas constan de cinco o seis años. A las primeras se les denomina estudios de primer ciclo y con las segundas se completa el segundo ciclo.
}

Rev. Diálogo Educ., Curitiba, v. 8, n. 23, p. 39-54, jan./abr. 2008 
de 6 de agosto de 1970, Ley General de Educación) en su artículo 102.2 b) se señalaba que: "Los profesores de Bachillerato, de las Escuelas Universitarias y de Formación del Profesional, la [formación la] obtendrán después de la titulación científica respectiva, mediante cursos intensivos dados por los Institutos de Ciencias de la Educación."

En la discusión que se realizó en torno a esa ley se indicaba que la duración de esos cursos debería ser de "seis meses o un cuatrimestre ya que no se debían prolongar los estudios de manera indefinida". Este apartado de la ley se desarrolla con posterioridad en la Orden de 8 de julio de 1971 en la que se establece que el curso en cuestión debería tener dos ciclos: en el primero, de carácter teórico y con una duración de 150 horas, se estudiarían los principios generales de la educación en torno a las temáticas siguientes:

1. principios, objetivos y problemática de la educación en sus aspectos psicológicos, sociológicos e históricos;

2. tecnología y sistemas de innovación educativa;

3. didácticas especiales.

El segundo ciclo sería de carácter práctico y consistiría en el ejercicio de la labor docente en los centros de secundaria bajo la supervisión de dos o tres Profesores tutores durante otras 150 horas.

Este curso ha sufrido algunas modificaciones desde entonces, prácticamente regidas por el sentido común más que por disposiciones oficiales, y a pesar de que la formación pedagógica del profesorado de secundaria se considera muy deficiente, las diferentes reformas que se han propuesto desde entonces, por unas causas u otras (generalmente económicas) no han cuajado en el sistema educativo. Por eso, cada universidad y algunas Comunidades Autónomas han ido adaptando los estudios para equipararlos al sistema educativo vigente, pero a todas luces han quedado obsoletos.

Así, aun cuando los profesores de educación secundaria son todos licenciados, a lo largo de su carrera no han recibido ninguna formación didáctica salvo en algunas Universidades en las que partir de la buena voluntad, disponibilidad, insistencia y convencimiento de los profesores del área de didáctica de la matemática se han creado asignaturas, en algunos casos optativas, en otros de libre disposición, que intentan ir creando la conciencia de la necesidad de estas materias en la formación de los profesionales de la educación.

8 Los Institutos de ciencias de la Educación se crearon en el año 1970 en el seno de las universidades con el objetivo de la formación del profesorado de todo el sistema educativo.

Rev. Diálogo Educ., Curitiba, v. 8, n. 23, p. 39-54, jan./abr. 2008 
El último intento para dotar a la formación del profesorado de Enseñanza Secundaria de un carácter profesional, se produjo con motivo de la aprobación de la Ley de Reforma Universitaria (LRU) en 1983. El Gobierno Central creó una serie de Grupos de expertos para que emitiesen Informes sobre la reforma de los Planes de Estudio. Uno de los Grupos (el Grupo XV) se encargó de las titulaciones universitarias correspondientes a los distintos niveles del sistema educativo anteriores a la Universidad. La propuesta del Grupo XV diferenciaba dos titulaciones para el profesorado de educación secundaria: Profesor de Educación Secundaria Obligatoria y Profesor de Educación Secundaria Postobligatoria, en ambos casos con mención a una disciplina concreta. Estas enseñanzas se estructuraban como de Segundo Ciclo con una duración máxima de dos años y un número máximo de mil quinientas horas (150 créditos); se proponía que el acceso a estas titulaciones se hiciese después de haber cursado una Diplomatura universitaria de tres años, de entre las que se declarasen pertinentes a cada especialidad de profesorado. Las directrices sugeridas por el Grupo XV preveían materias comunes a todas las especialidades como Didáctica General del Ciclo, Organización del Centro Escolar, Psicología y Sociología de la Educación y Psicología del desarrollo, entre otras; y materias específicas de cada especialidad para el Título de Profesor de Enseñanza Secundaria Obligatoria. En la titulación de Profesor de Educación Secundaria Postobligatoria también aparecían unas materias troncales de contenido psicopedagógico y en cuanto a las Didácticas especiales el documento señalaba que se determinarían de acuerdo con las especialidades que se estableciesen en el Bachillerato, que en aquellas fechas estaba en estudio. En ambas titulaciones se incluía un periodo de Prácticas docentes (Practicum). Sin embargo, la Ponencia de Reforma de las Enseñanzas Universitarias del Consejo de Universidades, en un documento de fecha Abril de 1987 pero no sometido a discusión hasta Marzo de 1989, se opuso a la existencia de estas titulaciones alegando que se profesionalizaba en exceso estos estudios universitarios, y se rompía con la tradición.

La Ponencia sugería una contrapuesta por medio de la realización de un Curso de Capacitación Pedagógica (CCP), organizado por las diferentes Universidades, una vez finalizada la Licenciatura correspondiente. Esta alternativa fue aceptada por el Gobierno que debía publicar unas directrices generales. El CCP sólo se impartió en algunas Comunidades Autónomas, ${ }^{9}$ conviviendo, en algunas de ellas, con el antiguo Curso de Aptitud Pedagógica (CAP) emanado de la Ley General de Educación de 1970. Posteriormente, la Ley Orgánica de Educación (LOE) estableció el Título de Especialización Didáctica (TED), publicándose incluso el decreto que lo desarrollaba, pero el TED nunca fue impartido.

9 España está dividida en diferentes comunidades autónomas cada una de las cuales tiene transferidas competencias relativas a la educación y puede legislar teniendo presente el marco general establecido para toda la nación.

Rev. Diálogo Educ., Curitiba, v. 8, n. 23, p. 39-54, jan./abr. 2008 
Actualmente, la formación inicial de los profesores de enseñanza secundaria en España, está en proceso de reforma ya que según la actual L.O.E. (Ley Orgánica de la Educación) publicada en el B.O.E. de 4 de mayo de 2006 en los artículos 94 y 100 se indica:

Artículo 94: "Para impartir las enseñanza de educación secundaria obligatoria y de bachillerato será necesario tener el título de Licenciado, Ingeniero o Arquitecto, o el título de grado equivalente, además de la formación pedagógica y didáctica del nivel de posgrado..."

Artículo 100 "La formación inicial del profesorado de las diferentes enseñanzas reguladas en la presente ley se adaptarán al sistema de grados y posgrados del espacio europeo de educación superior según lo que establezca la correspondiente normativa básica".

\section{E1 proceso de convergencia europea (o de Bolonia)}

El proceso de convergencia europea impone que los programas de formación del profesorado deberán preparar profesores que estén capacitados para desempeñar su trabajo en cualquier estado de la Unión Europea, además de facilitar la movilidad de los estudiantes durante la realización de sus estudios superiores. Se pretende no sólo reformar los títulos sino lograr un modelo atractivo y exportable, una educación de calidad y al alcance de todos. La nueva estructura de educación superior se organiza en tres ciclos: Grado (240 ECTS), Máster (60 ECTS) y Doctor (60 ECTS de formación y tesis doctoral)

Para configurar estos planes de estudios se creo en España en el año 2003, la llamada "red de Magisterio" establecida como consecuencia de una convocatoria de la $\mathrm{ANECA}^{10}$ para incorporar los estudios de Magisterio a la normativa de Bolonia. Esta red, de la que formaban parte, prácticamente todos los directores de las Escuelas de Magisterio y los decanos de las Facultades de Educación (en total fueron 44 participantes) publicó, como consecuencia del trabajo realizado durante un año, ${ }^{11}$ el Libro Blanco de la Titulación de Magisterio, donde proponía dos

10 La ANECA es la Agencia Nacional de Evaluación y Acreditación creada en 2002 para contribuir a la mejora de la calidad del sistema de educación superior.

11 El trabajo que realizaron los decanos de las Facultades de Educación y los directores de las Escuelas de Magisterio fue bastante amplio e intenso cubriendo aspectos como: un análisis de los estudios de magisterio en Europa, la selección del modelo más adecuado, la demanda de los títulos y las plazas ofertadas en cada uno de los centros, la inserción laboral de los titulados durante el último quinquenio, el perfil profesional a partir de la opinión de 880 informantes procedentes de diferentes ámbitos relacionados con el magisterio, una valoración de las competencias generales a partir de una muestra de 180 profesores de 18 universidades, la enumeración, clasificación y valoración de las competencias específicas y el diseño final del título que incluía: la definición de los objetivos, la estructura general, la distribución de los contenidos y la asignación de créditos ECTS.

Rev. Diálogo Educ., Curitiba, v. 8, n. 23, p. 39-54, jan./abr. 2008 
titulaciones una de Maestro de Educación Infantil y otra de Maestro de Educación Primaria (con carácter generalista y al mismo tiempo especializado) con una duración de 240 créditos ECTS ${ }^{12}$ (cuatro cursos). Se propone un bloque de materias troncales (en torno al $70 \%$ de la titulación) y cuatro itinerarios formativos (Lengua Extranjera, Educación Física, Educación Musical y Educación Especial) especializados en el caso de la Educación Primaria. La formación práctica recibe 42 ECTS y las materias básicas del currículo pasan a tener un peso de 102 ECTS.

En cuanto a las materias se organizan en distintos bloques: Bloque de materias comunes (psico-socio-pedagógicas), Bloque de materias comunes de áreas del currículo (Matemáticas, Lengua, Ciencias, Geografía e Historia y Educación Artística-plástica), Bloque de materias específicas de cada itinerario (Educación Física, Educación Musical, Educación Especial o Lengua Extranjera), bloque de prácticas docentes (Prácticum) y créditos de libe disposición.

Posteriormente, durante el año 2006, el MEC elaboró unas fichas técnicas (a través de comisiones de expertos) para cada una de las titulaciones de grado que en relación con los títulos de maestros se reafirma en el establecimiento de dos títulos de grado: uno que conduce al título de magisterio en educación primaria y otro de educación infantil. La distribución de asignaturas y créditos en estos títulos sería la siguiente:

- un total de 240 ECTS;

- créditos de formación académica básica, 180 ECTS;

- formación adicional de orientación académica o profesional que debe superar el alumno: 60 ECTS de los cuales, al menos 40 créditos han de corresponder al Prácticum.

12 ECTS European Credit System Transfer es un sistema centrado en el estudiante, que se basa en la carga de trabajo del estudiante necesaria para la consecución de los objetivos de un programa Se estableció inicialmente para la transferencia de créditos: el sistema facilitaba el reconocimiento de los períodos de estudios en el extranjero, incrementando así la calidad y el volumen de la movilidad de los estudiantes en Europa. El ECTS se está convirtiendo ahora en un sistema de acumulación que podrá aplicarse a nivel institucional, regional, nacional y europeo. El ECTS se basa en la convención de que 60 créditos miden la carga de trabajo de un estudiante a tiempo completo durante un curso académico. La carga de trabajo para un estudiante en un programa de estudios a tiempo completo en Europa equivale, en la mayoría de los casos, a 1 500 o 1800 horas por año, y en tales casos un crédito representa de 25 a 30 horas de trabajo.

Rev. Diálogo Educ., Curitiba, v. 8, n. 23, p. 39-54, jan./abr. 2008 
TABLA 1 - CONTENIDOS FORMATIVOS COMUNES: 120 ECTS + 40 ECTS

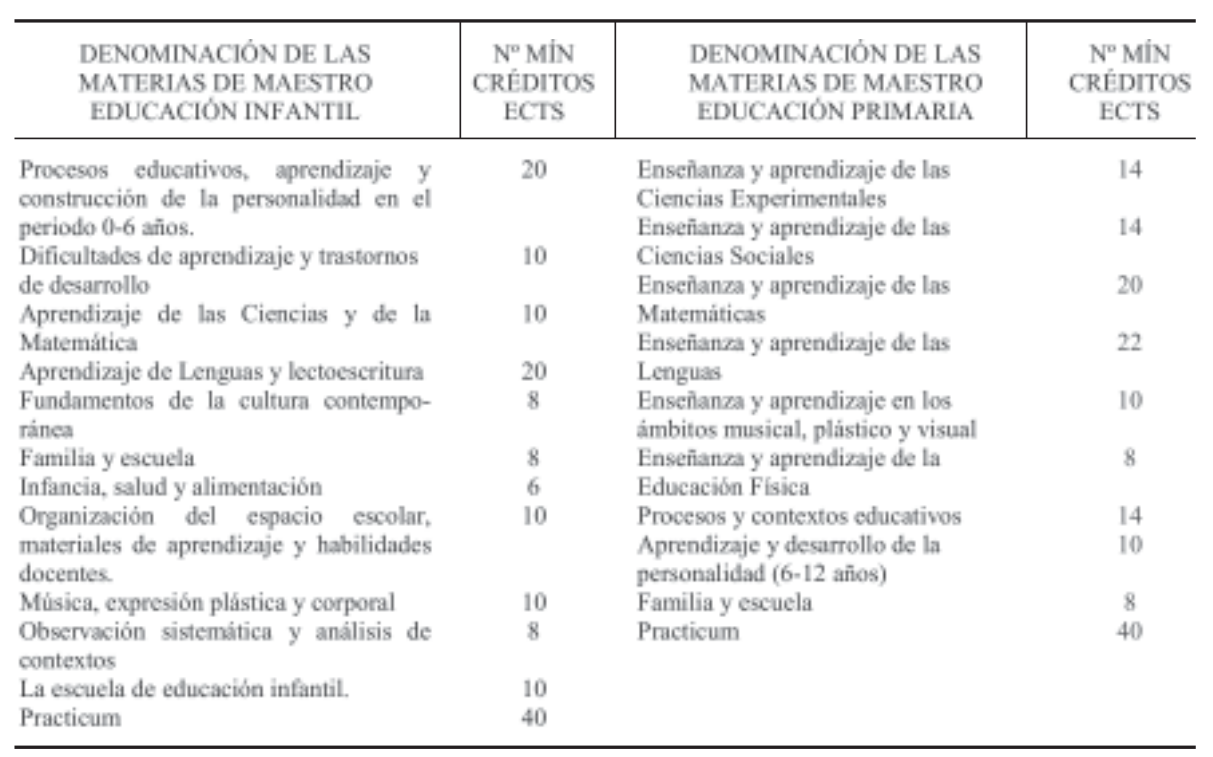

En cuanto a la formación de los profesores de educación secundaria en los libros Blancos de las distintas carreras, elaborados como consecuencia de diferentes convocatorias de la ANECA, en las nuevas titulaciones las competencias didácticas están prácticamente ausentes salvo algunas titulaciones como Matemáticas o Lengua Extranjera. Sin embargo, en las fichas técnicas elaboradas por el Ministerio no se hace referencia a ningún contenido didáctico en ninguna titulación.

Para completar la formación de un graduado como profesor de enseñanza secundaria se plantea un máster de 60 ECTS que contempla materias de índole general independientes de la titulación de la que provengan los futuros profesores (aprendizaje y desarrollo de la personalidad, procesos educativos y sociedad, familia y educación) y materias específicas relacionadas con las asignaturas que van a impartir (Formación disciplinar, el aprendizaje y la enseñanza de las materias de la especialidad y materias afines, innovación docente e iniciación a la investigación educativa). Las primeras sobre un total de 60 ECTS cubren un $30 \%$ del máster y las segundas un $50 \%$. Se valora positivamente el diseño de este posgrado por lo que supone de avance respecto de la situación tan precaria en la que se encuentra actualmente la formación de los profesores de secundaria, aunque ésta no sea la solución ideal.

Un aspecto que resulta ciertamente delicado es el correspondiente a la materia correspondiente al Practicum. Esta materia debería lograr una conexión eficaz entre los profesores y centros de Educación Secundaria y profesores y

Rev. Diálogo Educ., Curitiba, v. 8, n. 23, p. 39-54, jan./abr. 2008 
centros de Enseñanza Universitaria a través de los tutores de prácticas y los equipos de innovación docente. Esto está contemplado en el borrador del decreto de grado, en el que se indica que los profesores de educación secundaria podrán involucrarse en el máster siendo profesores asociados de universidad. Pero existe una gran imprecisión en algunas expresiones. En cuanto a los aspectos prácticos asociados a esta materia, la Comunidad Autónoma debería establecer los convenios y acuerdos pertinentes para asegurar la realización correcta del Practicum, así como definir las tareas de alumnos y tutores a lo largo de su realización.

En el momento actual se está a la espera de que el gobierno de la nación dicte la normativa legal a la que deben ajustarse todos los títulos de grado y, en especial, las directrices de las que deben emanar los nuevos títulos relativos a la formación de profesores.

\section{REFERÊNCIAS}

ANECA Libro Blanco: Título de grado en magisterio. Madrid: ANECA, 2004.

ESCOLANO, A. Las escuelas normales: siglo y medio de perspectiva histórica. Revista de Educación, Madrid, n. 269, p. 55-76, 1982.

GONZÁlEZ ASTUDillo, M. T.; PINTO, J. La formación de profesores de enseñanza secundaria en España y Méjico. In: AYMERICH, J. V.; VIVES, S. M. Matemáticas para el siglo XXI. Castellón: Universidad Jaume I, 2006. p. 207-214.

GONZÁLEZ PÉREZ, T. Trazos históricos sobre la formación de maestros. Revista Interuniversitaria de formación del profesorado, Zaragoza, v. 21, p. 175-198, 1994.

JUNTA DE CASTILLA Y LEÓN. Estudio de evaluación de las matemáticas en el sistema educativo de Castilla y León. Valladolid: Junta de Castilla y León, 2007.

MADRID, D. Expectativas y demandas del profesorado respecto a la futura licenciatura de magisterio. In: ROMERO, A.; GUTIÉRREZ, J.; CORIAT, M. (Ed.). La formación inicial del profesorado a luz de los nuevos retos de convergencia de las polítIcas de la unión europea. Granada: Universidad de Granada, 2003. p. 113-128.

MALDONADO, A. Los planes de estudio de magisterio del año 1991. Aula de innovación educativa, Barcelona, n.143-144, p. 24-27, jul./ago. 2005.

Rev. Diálogo Educ., Curitiba, v. 8, n. 23, p. 39-54, jan./abr. 2008 
PEREYRA-GARCÍA, M. A vueltas con la formación inicial del profesorado. En ROMERO, A.; GUTIÉRREZ, J.; CORIAT, M. (Ed.). La formación inicial del profesorado a luz de los nuevos retos de convergencia de las políticas de la unión europea. Granada: Universidad de Granada, 2003. p. 109-112.

PUELLES, M. Educación e ideología en la España contemporánea. (1767-1975). Madrid: Labor, 2004.

RICO, L.; SIERRA, M. Contexto y evolución histórica de la formación en matemáticas y su didáctica de los profesores de primaria. In: GIMÉNEZ, J.; LLINARES, S.; SÁNCHEZ, V. (Ed.). El proceso de llegar a ser un profesor de primaria: cuestiones desde la educación matemática. Granada: Comares, 1996. p. 39-62.

Recebido: 15/10/2007

Received: 10/15/2007

Aprovado: 05/11/2007

Approved: 11/05/2007

Rev. Diálogo Educ., Curitiba, v. 8, n. 23, p. 39-54, jan./abr. 2008 